CERN LIBRARIES, GENEVA

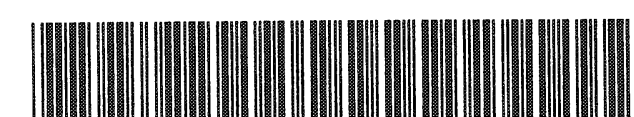

CM-PRS00001169

2667126 


\section{Antiprotons pack a greater punch}

Antiprotons should be four times better at destroying tumours than their antimatter equivalents - protons according to physicists at the CERN particle-physics laboratory in Geneva. The findings, due to be published in a forthcoming issue of the journal Radiotherapy and Oncology, could lead to new cancer-treatment techniques that minimize the damage done to healthy tissue surrounding tumours.

Protons are well suited to destroying tumour cells because they deposit most of their energy at a precise depth within the body. This depth is a function of proton energy, which can be set to minimize the energy absorbed by healthy tissue surrounding a tumour.

Antiprotons should deliver the same precision, but with an added benefit -when an antiproton nears the end of its journey, it will annihilate with a neutron or a proton, depositing additional energy and boosting the radiation dose delivered to the target area. Some of the fragments produced from this energy release could go on

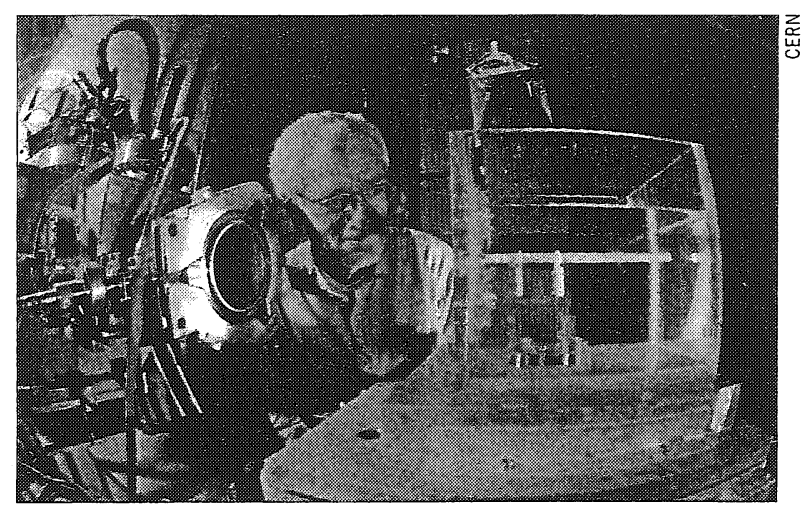

to destroy adjacent tumour cells.

ACE result

Researchers working on CERN's Michael Holzscheiter Antiproton Cell Experiment (ACE) at CERN's Antiproton have confirmed this hypothesis by Cell Experiment. irradiating hamster cells suspended in gelatin with beams of protons or antiprotons. The team compared the number of cells surviving within the target region - the tumour - to the number surviving within the area in front of the target region - healthy tissue. They concluded that antiprotons kill nearly four times as many target cells as protons, while intlicting the same damage on healthy tissue.

"We have taken the first step towards a novel treatment for cancer," says CERN physicist Michael Doser. "The results show that antiprotons are four times as effective as protons at terminating cells. Although it still has to be compared with other existing methods, it is a breakthrough in this area of investigation."

ACE spokesperson Michael Holzscheiter of the US company Pbar Labs LLC agrees. "To achieve the same level of damage to cells at the target area one needs four times as many antiprotons as protons. This significantly reduces the damage to the cells along the entrance channel of the beam for antiprotons compared with protons," he explains.

While initial results are certainly favourable, the researchers admit that clinical applications are still a decade or more away. One difficulty is that antiprotons can only be produced using high-energy particle accelerators. Currently, CERN's Antiproton Decelerator is the only facility in the world that can generate an antiproton beam of sufficiently low energy and high quality for this type of application. Tami Freeman 